\title{
Value of video monitoring for nocturnal seizure detection in a residential setting
}

M van der Lende ${ }^{1,2}$, FME Cox ${ }^{1}$, GH Visser ${ }^{1}$, JW Sander $^{1,3}$, RD Thijs $^{1,2,3}$

${ }^{1}$ Stichting Epilepsie Instellingen Nederland (SEIN), Achterweg 5, 2103 SW, Heemstede, The Netherlands

${ }^{2}$ Department of Neurology, Leiden University Medical Center (LUMC), Albinusdreef 2, 2333 ZA, Leiden, The Netherlands

${ }^{3}$ NIHR University College London Hospitals Biomedical Research Centre, Department of Clinical \& Experimental Epilepsy,

UCL Institute of Neurology, Queen Square, London WC1N 3BG, and Epilepsy Society, Chalfont St Peter, SL9 ORJ, UK

\section{Corresponding author:}

Dr Thijs, MD, PhD

Stichting Epilepsie Instellingen Nederland

Achterweg 5

2103 SW, Heemstede, The Netherlands

Phone 31-23-5588948

Fax 31-23-5588949

Email rthijs@sein.nl

Running title: Nocturnal video monitoring in epilepsy

\section{Key words:}

'Sudden Unexpected Death in Epilepsy', 'seizure', 'seizure detection', 'acoustic detection', 'bed motion sensor'

Number of text pages:

Number of words:

Number of references:

Number of figures:
Number of tables: 


\section{Summary}

Objective: Following a sudden death at a residential care unit, the Dutch Health and Care Inspectorate advised to intensify the use of video monitoring (VM) at the unit. We assessed whether VM resulted in increased identification of seizures requiring clinical intervention.

Methods: The unit provides care for 340 individuals with refractory epilepsy and severe learning disabilities. Acoustic detection systems (ADS) cover all, 37 people also have a bed motion sensor (BMS) and 46 people with possible nocturnal seizures are now monitored by VM. During a six month period, in all cases of a suspected seizure we asked the caregivers to specify which device alerted them and to indicate whether this led to an intervention. Staff costs of VM were estimated using payroll information.

Results: We identified 1208 seizures in 37 individuals; four had no nocturnal seizures; 393 (33\%) seizures were only seen on video. In 169 of 1208 (14\%) seizures an intervention was made and this included 39 of $393(10 \%)$ seizures only seen on video.

When compared to seizures observed with an ADS or BMS, seizures only seen on video were more often tonic seizures $(71 \%$ versus $22 \%, p<0.001)$ and occurred mostly in the beginning or at the end of the night ( $40 \%$ versus $26 \%, \mathrm{p}<0.001)$. The extra staff costs of monitoring was 7,035 euro per seizure only seen on video and leading to an intervention.

Significance: VM facilitates nocturnal surveillance, but the costs are high. This underscores the need for development of reliable seizure detection devices. 
Key words: 'Sudden Unexpected Death in Epilepsy', 'seizure', 'seizure detection', 'acoustic detection', 'bed motion sensor'

\section{Key point box:}

- $\quad$ Video monitoring is particularly helpful to detect tonic seizures.

- $\quad$ Video monitoring may also help to detect seizures late in the evening or in early morning, most likely due to background noise drowning out seizure noises, making acoustic detection systems less reliable

- Video monitoring may facilitate detection of seizures requiring intervention

- $\quad$ Video monitoring may be effective to detect clinically relevant seizures but costs are high. This emphasizes the need for reliable seizure detection devices 


\section{Introduction}

Sudden unexpected death in epilepsy (SUDEP) happens mostly after unwitnessed, nocturnal convulsions (1-3). Night supervision seems to be associated with a lower risk(4). At a boarding school for pupils with epilepsy all SUDEPs occurred when students were unsupervised (5) suggesting that surveillance is protective for highrisk populations.

Various seizures detection systems have been promoted, including acoustic devices, mattress sensors, video detection systems and wearables recording electrodermal activity, heart rate, muscle activity or movement (accelerometry)(6-9). It is, however, unclear which device can detect nocturnal seizures most accurately and whether this can reduce SUDEP risk $(10,11)$.

Following a SUDEP at our residential care facility, the Dutch Health and Care Inspectorate advised intensification of video monitoring (VM). It is likely that VM may facilitate seizure detection, but the clinical relevance is not established.

To quantify the benefits of additional nocturnal VM, we assessed whether nocturnal VM resulted in an increase in seizures requiring nursing intervention (e.g. emergency medication).

\section{Methods}

\section{Study population}

SEIN has a residential care facility housing 340 people with refractory epilepsy and severe learning disabilities. Residents are supported by care staff trained to 
recognize seizures and administrate rescue medication if required. Each resident has a seizure diary which is updated contemporaneously.

Various monitoring devices are used: all have an acoustic detection system (ADS) (DeHeerMedicom, Born, The Netherlands), and some have a bed motion sensor (BMS) (Epicare 3000, Danish Care Technology, Sorø, Denmark) or a video monitoring system (DeHeerMedicom, Born, The Netherlands). ADS and BMS detection thresholds are individually set.

There are six seizure monitoring units, each staffed with up to four caregivers. Each receives data from up to 80 people: up to 80 ADSs, up to 10 BMSs and up to 16 video feeds. One person monitors all systems in units with up to 12 video feeds. In units with over 12 video feeds, two monitor the systems. Roles are rotated every 1.5 hours. Those not on monitoring duty perform physical checks. When a seizure is suspected, the resident is contacted through an intercom system. If there is no response, a caregiver will determine if assistance is required.

After the Inspectorate advice, new criteria for VM were formulated. It was recommended for all with (1) putative evidence of unwitnessed nocturnal convulsions such as incontinence or a tongue bite on awakening and (2) convulsions in the late evening or early morning as the ADS is less reliable then due to background noise of people getting ready for bed / getting out of bed. Up to 80 ADSs are monitored by one person and sounds made by a subject can drown out seizure-related noises of another. The number of video-monitored residents increased from 12 to 46 , leading to an increment of night staff from 20 to 24 per shift.

All these 46 residents were asked to participate. Informed consent was obtained from or in some cases assent was obtained from legal guardians for those who
Commented [11]: This is not needed to understand the paper! Carers are trained!!!!
Commented [LMvd2]: 41 of 46 consented, so I can't say they all consented 
agreed to participate. Demographic data, medications, epilepsy syndrome, duration, seizure types, IQ and body mass index were extracted from the notes. Seizure frequencies were derived from the seizure diaries.

During a six-month period, caregivers recorded details of each nocturnal seizure in those monitored: time and type of seizure, detecting monitoring device, if the person was attended and if an intervention was required.

Caregivers used a similar seizure classification sheet to usual care including the following seizure types: convulsive, tonic, myoclonic, complex partial and unclassified. A nursing intervention was scored when the caregiver (1) repositioned the subject, (2) administered rescue medication, or (3) stimulated the vagal nerve stimulator. (see appendix A)

All data was collected into a database (SPSS for windows, version 22) and divided into two groups: seizures only seen on video and seizures detected by ADS or BMS, whether seen on video or not. These groups were then compared, looking for differences in seizure types, seizure timing and interventions performed. Actual staff costs were obtained from the appropriate department.

\section{Validation of caregiver reports}

If a seizure was suspected, staff pressed the record button thus saving ten minutes of video feed. A random sample of two seizures, per seizure type, per subject was selected of all seizures only seen on video to validate the caregivers' seizure classification. One neurologist from a panel (RDT, FC and GHV) blinded to the caregivers scores reviewed the videos. They recorded whether they agreed that the event was epileptic and classified the seizure type. They used the same seizure 
nomenclature as the caregivers, but were asked to specify further the event type (e.g. hyperkinetic seizure). To score the certainty of the seizure classification and epileptic nature of the event a score from 1 (not certain at all) to 5 (very certain) was used (See appendix B). All videos for which there were doubts over the nature or classification (score 3 or lower) were reviewed by all three neurologists to establish a consensus agreement.

BMS and ADS event logs were automatically stored. BMS logs were used to check whether seizures reported as being only seen on video had no matching BMS record. For the ADS a random sample of three nights with nocturnal events per subject was selected and checked for event logs of seizures reported as picked up by the ADS.

\section{Statistical analysis}

Differences between seizures only seen on video versus all other seizures were estimated by fitting a multivariable logistic regression model. To account for the correlation between seizures in the same individual we used generalized estimating equations (GEE). All variables were entered as predictors using a backwards selection procedure $(p<0.05)$ to determine which variables are independent determinants of seizures detected only with help of VM.

\section{Results}

Forty-one of 46 people monitored were included. Five declined participation. All had an ADS and fourteen a BMS. General characteristics are listed in table 1.

\section{Reported events}


During the six-month period caregivers reported 1260 events in 37 of the 41 participants. No seizures were identified in four. Fifty-two events were false positives as determined by the caregivers attending the individual.

An intervention occurred in 167 of 1208 seizures. Twelve of the 37 individuals with nocturnal seizures did not receive an intervention.

\section{Characteristics of seizures only seen on video}

Caregivers reported that $393(33 \%)$ seizures, in 29 of 37 people, were only seen on video. When compared to seizures observed with an ADS or BMS, seizures only seen on video occurred more often either at the beginning or end of the night $(41 \%$ versus $26 \%$ of seizures, $p<0.001)$ and were more often tonic seizures $(71 \%$ versus $22 \%$ of seizures, $p<0.001)$.

Convulsive seizures (CSs) and myoclonic seizures were also frequently observed with the ADS or BMS: 19 of 37 people had CSs seen only on video, but this was only $12 \%$ of all detected CSs (figure 1). Seven of these 19 people had a BMS and 19 of these 46 convulsive seizures (41\%) occurred either early morning or late evening.

Of the 393 seizures only seen on video, 39 required an intervention (table 2) and 14 of 29 people with seizures only seen on video had an event requiring an intervention. When compared to seizures only seen on video, seizures identified on an ADS or BMS required more interventions ( $16 \%$ versus $10 \%, p=0.006$ ).

When fitting a multivariable logistic regression model using generalized estimating equations, only three variables (seizure time, seizure type and intervention) were significant independent predictors. (table 3) 


\section{Validation of caregivers' reports}

We randomly selected 89 videos of events only seen on video; 26 were excluded, as recording started too late in the seizure course to allow for a definite assumption. The remaining 63 were classified by the panel.

There was agreement on the epileptic nature of the event in 58 of 63 videos (92\%). There were some differences in the caregivers' classification compared with those of the panel. Seizures classified as CSs by the caregivers were often categorized by the panel as focal hyperkinetic seizures.

BMS logs were reviewed for 161 seizures only seen on video as reported by the caregivers. In 134 (83\%) it was confirmed that the BMS did not sound in the 15 minutes adjacent to the reported seizure onset.

If caregivers reported that the ADS alerted them, an event could be identified in the $\log$ in 76 of 82 (93\%) seizures between 15 minutes prior to and after the reported seizure onset.

\section{Cost-effectiveness}

Due to the increase in people video-monitored, four extra staff were required per night, resulting in extra yearly personnel costs of $€ 548,762$ ( $€ 274,381 / 6$ months). We identified 393 seizures which were only seen with VM: 274,381/393=€698 per detected seizure. Thirty-nine seizures receiving an intervention were only seen on video: $274,381 / 39$ seizures $=€ 7,035$ per seizure and 47 CSs were only seen on video: $274,381 / 47=€ 5,838$ per seizure. 


\section{Discussion}

VM in conjunction with ADS and BMS facilitated nocturnal surveillance: $33 \%$ of all observed seizures were only seen on video. VM also helped detecting clinically relevant seizures: of all only seen on video, $10 \%$ required an intervention. Seizure timing (late night or early morning) and seizure type (tonic seizures) were significantly associated with seizures only seen on video. The added value of VM should, however, be weighed against extra costs.

We used the caregivers' reports as gold standard to determine which device alerted them to a seizure. This has implications as we cannot guarantee that all seizures were noticed. Those with subtle signs are likely missed but also those with obvious motor signs may have been ignored, as caregivers had to continuously pay attention to multiple video feeds and other detection devices. While we acknowledge that we may have underestimated the number of seizures, we did not consider screening videos of reported seizure free periods, as we aimed to assess the added value of VM and not to quantify its (obvious) limitations. Such exercise would also require additional EEG monitoring. A study on a similar population reported that when using a combination of video-EEG and accelerometry, the number of detected seizures was seven-fold higher than seizures seen by nurses. (6).

Caregivers indicated which device captured a seizure. Multitasking may also have resulted in other alarms being ignored. We crosschecked caregivers' reports with ADS and BMS event logs and agreement was good: $93 \%$ for the ADS and $83 \%$ for the BMS. For the ADS, though, we could not ascertain whether seizures only seen on video were truly silent: the system records an event for any sound above the threshold. Other sounds in the peri-ictal period (e.g. door shutting) may also result in 
a data point. The same principle applies to BMSs: an alarm signal may also result from a subject repositioning after a seizure. No events were found in $83 \%$ of seizures without a staff record of a BMS alarm.

We also relied on caregivers' seizure classification. An expert panel, therefore, evaluated a random seizures subsection. We found a high agreement (92\%) on the epileptic nature of an event between the panel and caregivers. Agreement on seizure type classification was, however, poor (38\%), confirming previous report (12). The caregivers' most frequent inaccuracy was classifying "hyperkinetic focal seizures" as CSs. Detection systems might have the same limitation as caregivers judgment: a BMS will not allow differentiation between frontal lobe seizures and CSs. In view of the classification errors we may have overestimated the number of CSs that were detected by video only.

The majority of seizures only seen on video were tonic seizures. BMSs are likely to miss seizures without excessive movement: a study on a BMS reported that it only identified three of eleven tonic seizures on an awake subject and none while asleep(13). A high number of seizures only seen on video were either on late evening or early morning. ADSs are probably less reliable during periods of high background noise.

We found the greatest added value of VM to be for tonic seizures, but there is no evidence that these increase SUDEP risk. SUDEP was preceded by a CS in all monitored cases.(14) Case control studies show a high CS frequency to be a major SUDEP risk factor (4, 15-17). Monitoring devices designed to detect nocturnal CSs may therefore decrease a person's SUDEP risk: people are less likely to die of 
SUDEP when they share a room or when there is a listening device(4). An ADS is probably a sensitive way to detect CSs, as in $85 \%$ of CSs an ictal cry is heard (18).

Detecting a CS that could be followed by SUDEP is no guarantee for preventing SUDEP. There are several reports of observed SUDEP cases, where a witness could not prevent it (19) and prompt resuscitation procedures failed (11). We are aware of two (unpublished) cases of residents dying of SUDEP despite VM.

\section{Clinical implications}

VM appeared very costly: personnel outlays were estimated at $€ 7,035$ per seizure seen only on video and requiring an intervention. With SUDEP estimated to occur in 1 of every $2,000-5,000$ CSs (20), it would costs millions to detect an additional seizure leading to SUDEP, without guarantee that this will be preventive. We believe that the limited added value of VM is outweighed by the high costs. VM might facilitate detection of CSs as well, but this seemed often related to ADS failure or BMS absence. We thus do not recommend widespread VM implementation. In view of high costs and questionable protective effects, it seems more reasonable to optimize ADS or to consider other seizure detection devices. Our study underscores the need for the development of less costly, reliable detection devices. As those with intellectual disabilities have a higher seizure burden and SUDEP risk, the search for protective measures is even more urgent in this population $(17,21-23)$.

\section{Acknowledgments}

The authors are grateful to $\mathrm{Dr}$ GS Bell for reviewing the manuscript and to $\mathrm{R}$ Straathof for his help with collection of the data. JWS is based at the UCLH/UCL 
Comprehensive Bio-Medical Research Centre which received a proportion of funding from the Department of Health's NIHR Biomedical Research Centres funding scheme.

\section{Disclosures}

MvdL reports no disclosures. FMC reports no disclosures. GHV reports no disclosures. JWS receives research support from the Dr. Marvin Weil Epilepsy Research Fund, Eisai, GSK, WHO, and EU FP7 and has been consulted by and received fees for lectures from GSK, Eisai, Lundbeck, Teva and UCB. RDT receives research support from the Dutch National Epilepsy Fund, NUTS Ohra Fund, Medtronic, and AC Thomson Foundation, and has received fees for lectures from Medtronic, UCB and GSK.

\section{Ethical Publication Statement}

We confirm that we have read the Journal's position on issues involved in ethical publication and affirm that this report is consistent with these guidelines. 
Van der Lende 14

Figure 1: Seizures only seen on video, versus seizures observed using the ADS or BMS.

$A D S$ acoustic detection system; BMS bed motion sensor 


\section{References}

1. Nashef L, Garner S, Sander JW, Fish DR, Shorvon SD. Circumstances of death in sudden death in epilepsy: interviews of bereaved relatives. Journal of neurology, neurosurgery, and psychiatry. 1998;64(3):349-52. Epub 1998/04/04.

2. Nobili L, Proserpio P, Rubboli G, Montano N, Didato G, Tassinari CA. Sudden unexpected death in epilepsy (SUDEP) and sleep. Sleep medicine reviews. 2011;15(4):237-46. Epub 2010/10/19.

3. Lamberts RJ, Thijs RD, Laffan A, Langan Y, Sander JW. Sudden unexpected death in epilepsy: people with nocturnal seizures may be at highest risk. Epilepsia. 2012;53(2):253-7. Epub 2011/12/24. 4. Langan Y, Nashef L, Sander JW. Case-control study of SUDEP. Neurology. 2005;64(7):1131-3. Epub 2005/04/13.

5. Nashef L, Fish DR, Garner S, Sander JW, Shorvon SD. Sudden death in epilepsy: a study of incidence in a young cohort with epilepsy and learning difficulty. Epilepsia. 1995;36(12):1187-94. Epub 1995/12/01.

6. Nijsen TM, Arends JB, Griep PA, Cluitmans PJ. The potential value of three-dimensional accelerometry for detection of motor seizures in severe epilepsy. Epilepsy \& behavior : E\&B. 2005;7(1):74-84. Epub 2005/06/25.

7. Kalitzin S, Petkov G, Velis D, Vledder B, Lopes da Silva F. Automatic segmentation of episodes containing epileptic clonic seizures in video sequences. IEEE transactions on bio-medical engineering. 2012;59(12):3379-85. Epub 2012/09/06.

8. Van de Vel A, Cuppens K, Bonroy B, Milosevic M, Jansen K, Van Huffel S, et al. Non-EEG seizure-detection systems and potential SUDEP prevention: state of the art. Seizure. 2013;22(5):34555. Epub 2013/03/20.

9. Ramgopal S, Thome-Souza S, Jackson M, Kadish NE, Sanchez Fernandez I, Klehm J, et al. Seizure detection, seizure prediction, and closed-loop warning systems in epilepsy. Epilepsy \& behavior : E\&B. 2014;37:291-307. Epub 2014/09/01.

10. Ryvlin P, Nashef L, Tomson T. Prevention of sudden unexpected death in epilepsy: a realistic goal? Epilepsia. 2013;54 Suppl 2:23-8. Epub 2013/05/10.

11. Swinghamer J, Devinsky O, Friedman D. Can post-ictal intervention prevent sudden unexpected death in epilepsy? A report of two cases. Epilepsy \& behavior : E\&B. 2012;24(3):377-9. Epub 2012/06/02.

12. Whitten $E$, Griffiths A. Implementing epilepsy guidelines within a learning disability service. Seizure. 2007;16(6):471-8. Epub 2007/05/01.

13. Poppel KV, Fulton SP, McGregor A, Ellis M, Patters A, Wheless J. Prospective Study of the Emfit Movement Monitor. Journal of child neurology. 2013;28(11):1434-6. Epub 2013/02/01.

14. Ryvlin P, Nashef L, Lhatoo SD, Bateman LM, Bird J, Bleasel A, et al. Incidence and mechanisms of cardiorespiratory arrests in epilepsy monitoring units (MORTEMUS): a retrospective study. The Lancet Neurology. 2013;12(10):966-77. Epub 2013/09/10.

15. Hesdorffer DC, Tomson T, Benn E, Sander JW, Nilsson L, Langan Y, et al. Do antiepileptic drugs or generalized tonic-clonic seizure frequency increase SUDEP risk? A combined analysis. Epilepsia. 2012;53(2):249-52. Epub 2011/12/24.

16. Nilsson L, Farahmand BY, Persson PG, Thiblin I, Tomson T. Risk factors for sudden unexpected death in epilepsy: a case-control study. Lancet (London, England). 1999;353(9156):88893. Epub 1999/03/27.

17. Walczak TS, Leppik IE, D'Amelio M, Rarick J, So E, Ahman P, et al. Incidence and risk factors in sudden unexpected death in epilepsy: a prospective cohort study. Neurology. 2001;56(4):519-25. Epub 2001/02/27.

18. Elzawahry H, Do CS, Lin K, Benbadis SR. The diagnostic utility of the ictal cry. Epilepsy \& behavior : E\&B. 2010;18(3):306-7. Epub 2010/07/16. 
19. Langan Y, Nashef L, Sander JW. Sudden unexpected death in epilepsy: a series of witnessed deaths. Journal of neurology, neurosurgery, and psychiatry. 2000;68(2):211-3. Epub 2000/01/25.

20. Shorvon SD. Handbook of Epilepsy Treatment. 3 ed: John Wiley \& Sons, 2010; 2010.

21. Kiani R, Tyrer F, Jesu A, Bhaumik S, Gangavati S, Walker G, et al. Mortality from sudden unexpected death in epilepsy (SUDEP) in a cohort of adults with intellectual disability. Journal of intellectual disability research : JIDR. 2014;58(6):508-20. Epub 2013/05/08.

22. Young C, Shankar R, Palmer J, Craig J, Hargreaves C, McLean B, et al. Does intellectual disability increase sudden unexpected death in epilepsy (SUDEP) risk? Seizure. 2015;25:112-6. Epub 2014/12/03.

23. Devinsky O, Asato M, Camfield P, Geller E, Kanner AM, Keller S, et al. Delivery of epilepsy care to adults with intellectual and developmental disabilities. Neurology. 2015;85(17):1512-21. Epub 2015/10/02. 


\begin{tabular}{|c|c|}
\hline Age (years) & 37 (SD 18.3) \\
\hline Gender & $\begin{array}{r}24 \text { male / } 17 \\
\text { female }\end{array}$ \\
\hline \multicolumn{2}{|l|}{ Epilepsy etiology: } \\
\hline Genetic & $18(44 \%)$ \\
\hline Structural metabolic & $11(27 \%)$ \\
\hline Unknown & $12(29 \%)$ \\
\hline \multicolumn{2}{|l|}{ Seizure type: } \\
\hline Focal & $29(71 \%)$ \\
\hline Generalized & $12(29 \%)$ \\
\hline Age of onset (years) & 4.3 (SD 4.5) \\
\hline Duration epilepsy (years) & 33.1 (SD 17.7) \\
\hline \multicolumn{2}{|l|}{$\begin{array}{l}\text { Seizure type (number of } \\
\text { subjects): }\end{array}$} \\
\hline Tonic seizures & $20(48.8 \%)$ \\
\hline Convulsive seizures & $37(90.2 \%)$ \\
\hline Complex partial seizures & $22(53.7 \%)$ \\
\hline \multicolumn{2}{|l|}{ Daily seizure frequency } \\
\hline $0-4$ seizures / month & $26(63 \%)$ \\
\hline 5 - 9 seizures / month & $6(15 \%)$ \\
\hline$\geq 10$ seizures / month & $9(22 \%)$ \\
\hline \multicolumn{2}{|l|}{ Nocturnal seizure frequency } \\
\hline $0-4$ seizures / month & $31(76 \%)$ \\
\hline 5 - 9 seizures / month & $7(17 \%)$ \\
\hline$\geq 10$ seizures / month & $3(7 \%)$ \\
\hline \multicolumn{2}{|l|}{ Number of AEDs } \\
\hline none & $2(5 \%)$ \\
\hline $1 \mathrm{AED}$ & $5(12 \%)$ \\
\hline 2 AEDs & $11(27 \%)$ \\
\hline 3 AEDs & $15(37 \%)$ \\
\hline 4 AEDs & $8(20 \%)$ \\
\hline Vagal nerve stimulator & $7(17 \%)$ \\
\hline \multicolumn{2}{|l|}{$\mathrm{IQ}$} \\
\hline$\leq 20$ & $9(22 \%)$ \\
\hline $21-40$ & $6(15 \%)$ \\
\hline $41-60$ & $1024 \%)$ \\
\hline $61-80$ & $4(10 \%)$ \\
\hline$>80$ & $1(2 \%)$ \\
\hline unknown & $11(27 \%)$ \\
\hline Body Mass Index & 24.2 (SD 5.8) \\
\hline
\end{tabular}

Table 1: Patient characteristics $(\mathrm{N}=41)$

$A E D$ Anti-epileptic drug 
Van der Lende 18

\begin{tabular}{|l|r|r|}
\hline & $\begin{array}{r}\text { Seizures only seen } \\
\text { on video }(\mathrm{n}=393)\end{array}$ & $\begin{array}{r}\text { Seizures observed with } \\
\text { ADS or BMS }(\mathrm{n}=815)\end{array}$ \\
\hline All interventions & $39(9.9 \%)$ & $128(15.7 \%)$ \\
\hline $\begin{array}{l}\text { Repositioning the } \\
\text { person }\end{array}$ & $14(3.6 \%)$ & $6(7.1 \%)$ \\
\hline Activating VNS & $6(1.5 \%)$ & $59(7.2 \%)$ \\
\hline $\begin{array}{l}\text { Emergency } \\
\text { medication }\end{array}$ & $17(4.3 \%)$ & $5(0.6 \%)$ \\
\hline $\begin{array}{l}\text { VNS activation } \\
\text { and emergency } \\
\text { medication }\end{array}$ & $2(0.5 \%)$ & \\
\hline
\end{tabular}

Table 2: Interventions

$V M$ video monitoring, VNS vagal nerve stimulator 
Van der Lende 19

\begin{tabular}{|r|c|c|c|c|c|}
\hline & $\begin{array}{c}\text { Only seen } \\
\text { on VM ( } \mathrm{n}= \\
393)\end{array}$ & $\begin{array}{c}\text { Seen on an } \\
\text { ADS or BMS } \\
(\mathrm{n}=815)\end{array}$ & p-value & OR & 95\%Cl OR \\
\hline Seizure type: & & & & & \\
\hline Other & $115(29 \%)$ & $632(78 \%)$ & & & \\
\hline Time of seizure: & & & & & \\
\hline $22.30-0.00 / 6.30-8.30$ & 160 & 214 & $<0.001$ & 1.53 & $1.25-1.87$ \\
\hline hours & 160 & 601 & & & \\
\hline $0.00-6.30$ hours & 233 & & & & \\
\hline Intervention done: & & & & & \\
\hline no & 354 & 687 & 0.005 & 0.61 & $0.44-0.86$ \\
\hline yes & 39 & 128 & & & \\
\hline
\end{tabular}

Table 3: Seizures only seen on VM versus all other seizures. P-value and odds ratios calculated using a multivariable model in generalized estimating equations. $V M$ videomonitoring; $A D S$ acoustic detection system; $B M S$ bed motion sensor; $O R$ odds ratio 